\title{
Understanding microbial arsenic-mobilization in multiple aquifers: insight from DNA and RNA analyses
}

Wei Xiu ${ }^{1,2,3}$, Tiantian $\mathrm{Ke}^{3}$, Jonathan R. Lloyd ${ }^{4}$, Hokyung Song ${ }^{4}$, Jiaxing Shen ${ }^{3}$, Naji M. Bassil $^{4}$, David A. Polya ${ }^{4}$, Yi Zhao ${ }^{3}$, Huaming Guo ${ }^{1,3, *}$

${ }^{1}$ State Key Laboratory of Biogeology and Environmental Geology, China University of Geosciences, Beijing 100083, P.R. China

${ }^{2}$ Institute of Earth sciences, China University of Geosciences (Beijing), Beijing 100083, P.R. China

${ }^{3}$ School of Water Resources and Environment, China University of Geosciences (Beijing), Beijing 100083, P.R. China

${ }^{4}$ Williamson Research Centre for Molecular Environmental Science, School of Earth and Environmental Sciences, the University of Manchester, Manchester, United Kingdom M13 9PL, United Kingdom

* Corresponding author: Huaming Guo

China University of Geosciences (Beijing), Beijing 100083, P. R. China

E-mail: hmguo@cugb.edu.cn (H. Guo). Tel.: +86-10-8232-1366. Fax: +86-10-8232-1081.

(17 pages, 10 Figures, and 2 Tables) 


\section{Groundwater hydrogeochemical analysis}

2 All chemical samples were filtered using $0.45-\mu \mathrm{m}$ membrane and divided into sub-samples: an

3 acidified sub-sample containing ultrapure $6 \mathrm{M} \mathrm{HNO}_{3}$ to $\mathrm{pH}<2.0$ was prepared for major cation

4 and trace element measurements; a 0.25M EDTA preserved sub-sample was used for As

5 species analysis; another subsample containing concentrated $\mathrm{H}_{2} \mathrm{SO}_{4}$ to $\mathrm{pH}<2$ was prepared for

6 measurement of dissolved organic carbon (DOC); and another subsample with no additions

7 was prepared for anion analysis. All the subsamples were kept and transported to the laboratory

8 on ice, and then kept in a refrigerator at $4{ }^{\circ} \mathrm{C}$ until analysis within seven days.

9 A multiparameter portable meter (HI 9828, HANNA) was used to monitor water temperature,

$10 \mathrm{EC}, \mathrm{pH}$, and ORP through an in-line flow cell under minimal atmospheric contact after calibrated using standard solutions. During sampling, $\mathrm{Fe}^{2+} \mathrm{NH}_{4}{ }^{+}, \mathrm{NO}_{2}{ }^{-}$and $\mathrm{H}_{2} \mathrm{~S}$ concentrations were determined by using a portable spectrophotometer (DR2800, HACH), and alkalinity using a Model 16900 digital titrator $(\mathrm{HACH})$. Subsamples for trace element and cation analysis, anion analysis, As species analysis, and dissolved organic carbon (DOC) analysis were taken in-situ and determined in the laboratory. Concentrations of major cations and trace elements were determined by Inductively Coupled Plasma-Atomic Emission Spectroscopy (ICP-AES) and Inductively Coupled Plasma-Mass Spectroscopy (ICP-MS), respectively. The analytical accuracy of ICP-AES and ICP-MS for Fe and Mn was $\pm 3.0 \%$. The detection limit for As was $0.01 \mu \mathrm{g} / \mathrm{L}$. Unacidified aliquots were analyzed for major anions by ion chromatography (DX120, Dionex), with the analytical precision generally better than 5.0\%. For all samples, ion charge imbalances were less than $\pm 5 \%$. Arsenic species in groundwater samples were analyzed by HPLC-HG-AFS (AFS-9130, Titan), with a relative standard deviation (RDS) $< \pm 5 \%$ and analytical precision of $2.0 \%$. HPLC-HG-AFS was selected for arsenic speciation analysis because of its availability and because of the widespread successful usage of such coupled, hydride generation and atomic fluorescence spectrometry techniques including for the analysis 
26 of both inorganic and organic arsenicals ${ }^{1-3}$. The separation of As species was performed in a

27 Hamilton PRP-X100 (Hamilton, Reno, NV, USA) anion-exchange column $(250 \mathrm{~mm} \times 4.1 \mathrm{~mm}$, $10 \mu \mathrm{m})$ with $\mathrm{NH}_{4} \mathrm{H}_{2} \mathrm{PO}_{4}$ based mobile phase $(\mathrm{pH}=6)$. Detection limits of $\mathrm{As}(\mathrm{III})$ and $\mathrm{As}(\mathrm{V})$ were 2 and $4 \mu \mathrm{g} / \mathrm{L}$, respectively. DOC concentrations were determined using a Shimadzu TOCVCPH analyser (detection limit, $4 \mu \mathrm{gC} / \mathrm{L}$ ). The organic arsenic species (monomethyl- and dimethylarsenic, MMA and DMA) were below detection limits $(<2.0 \mu \mathrm{g} / \mathrm{L})$, which is consistent with previous study ${ }^{4}$.

\section{Sediment hydrogeochemical analysis}

Wet sediment samples were used for scanning electron microscopy (SEM), and to measure the Fe species $\left(\mathrm{TFe}_{\text {sed }}\right.$ and $\mathrm{Fe}(\mathrm{II})_{\text {sed }}$ ), water extractable ammonium (ammoniumextract.), nitrate (nitrate $e_{\text {extract. }}$ ) and sulfate (sulfate $e_{\text {extract.) }}{ }^{5}$. Sediment sub-samples were freeze-dried, disaggregated and then milled ( $<200$ mesh) and were used for X-ray diffraction spectra (XRD), total organic carbon (TOC) analysis, diffuse spectral reflectance $(530-520 \mathrm{~nm})^{6}$, and aciddigestion element analysis $\left(\mathrm{HNO}_{3}, \mathrm{HF} \text { and } \mathrm{HClO}_{4}\right)^{7}$. For the SEM, samples were prepared according to Zeng et al. $(2016)^{8}$ and determined using a Zeiss Supra 35VP SEM at an accelerating voltage of 3-10 kV, equipped with energy dispersive spectroscopy (EDS). For measurement of water extractable ammonium, nitrate and sulfate, $1 \mathrm{~g}$ wet sediment was mix with $10 \mathrm{~mL}$ ultra-pure water. was suspended with $10 \mathrm{~mL}$ of ultra-pure water and shaken for 30 $\min$ at $4^{\circ} \mathrm{C}$. After centrifugation at $250 \times \mathrm{g}$ for $5 \mathrm{~min}$ at $4^{\circ} \mathrm{C}$, the supernatant was diluted with ultra-pure water, and filtered through $0.2 \mu \mathrm{m}$ cellulose acetate filters and the solution was analyzed with a DX-120 Ion Chromatography (Dionex) ${ }^{5}$. TOC content was determined with a total organic carbon analyser (Multi N/C 2100S, Analytikjena, Germany). Sediment samples for major element analysis were air dried and grinded to less than 200 mesh and then determined using an X-ray fluorescence equipment (ARL AdvantXP+, Thermo). Concentrations of trace element were measured by a inductively coupled plasma mass 
51 spectrometry (ICP-MS, 7500C, Agilent) after acid $\left(\mathrm{HNO}_{3}, \mathrm{HF}\right.$ and $\left.\mathrm{HClO}_{4}\right)$ digestion ${ }^{7}$. A spectrometer (CM-700d, Konica Minolta) was used to detect the variation in diffuse spectral reflectance between $530 \mathrm{~nm}$ and $520 \mathrm{~nm}$, which can indicate the color of sediments and reflect the relative redox condition ${ }^{6}$. The X-ray diffraction (XRD) analysis was determined using a URD-6 powder diffractometer (Cu K-alpha source radiation).

DNA and RNA extraction, cDNA synthesis, sequencing, and data analysis

The raw reads were processed following the Mothur MiSeq SOP (https://www.mothur.org/wiki/MiSeq_SOP) using Mothur v.1.42.3 ${ }^{9}$. Chimeric sequences were removed using the VSEARCH v2.13.3 algorithm and sequences with homopolymers (> $6 \mathrm{bp)}$ were also removed. Sequences were clustered into operational taxonomic units (OTUs) based on $99 \%$ similarity using OptiClust algorithm ${ }^{10}$ and representative sequences for each OTUs were selected based on sequence abundance. Sequence reads were classified based on SILVA database v.132 ${ }^{11}$. Sequence reads classified as "Chloroplast”, “unknown”, "Archaea”, "Eukaroyta", and "Mitochondria" were removed.

\section{References}

(1) Maher, W. A.; Ellwood, M. J.; Krikowa, F.; Raber, G.; Foster, S. Measurement of

(2) Polya, D. A.; Watts, M. J. Sampling and Analysis for Monitoring Arsenic in Drinking Water. Best Practice Guide on the Control of Arsenic in Drinking Water. IWA Publishing July 15, 2017, p 0. https://doi.org/10.2166/9781780404929_049. 
for Arsenic Speciation. Appl. Organomet. Chem. 1998, 12 (6), 439-447. https://doi.org/https://doi.org/10.1002/(SICI)1099-0739(199806)12:6<439::AIDAOC718>3.0.CO;2-8

(4) Guo, H.; Zhou, Y.; Jia, Y.; Tang, X.; Li, X.; Shen, M.; Lu, H.; Han, S.; Wei, C.; Norra, S.; et al. Sulfur Cycling-Related Biogeochemical Processes of Arsenic Mobilization in the Western Hetao Basin, China: Evidence from Multiple Isotope Approaches. Environ. Sci. Technol. 2016, 50 (23), 12650-12659. https://doi.org/10.1021/acs.est.6b03460.

(5) Ihara, H.; Hori, T.; Aoyagi, T.; Takasaki, M.; Katayama, H. Y. Sulfur-Oxidizing Bacteria Mediate Microbial Community Succession and Element Cycling in Launched Marine Sediment. Front. Microbiol. 2017, 8 (FEB), 1-11. https://doi.org/10.3389/fmicb.2017.00152.

(6) Horneman, A.; Van Geen, A.; Kent, D. V.; Mathe, P. E.; Zheng, Y.; Dhar, R. K.; O’Connell, S.; Hoque, M. A.; Aziz, Z.; Shamsudduha, M.; et al. Decoupling of As and Fe Release to Bangladesh Groundwater under Reducing Conditions. Part I: Evidence from Sediment Profiles. Geochim. Cosmochim. Acta 2004, 68 (17), 3459-3473. https://doi.org/10.1016/j.gca.2004.01.026.

(7) Matthews, A.; Morgans-Bell, H. S.; Emmanuel, S.; Jenkyns, H. C.; Erel, Y.; Halicz, L. Controls on Iron-Isotope Fractionation in Organic-Rich Sediments (Kimmeridge Clay, Upper Jurassic, Southern England). Geochim. Cosmochim. Acta 2004, 68 (14), 31073123. https://doi.org/10.1016/j.gca.2004.01.019.

(8) Zeng, Q.; Dong, H.; Zhao, L.; Huang, Q. Preservation of Organic Matter in Nontronite against Iron Redox Cycling. Am. Mineral. 2016, 101 (1), 120-133. https://doi.org/10.2138/am-2016-5410.

(9) Schloss, P. D.; Westcott, S. L.; Ryabin, T.; Hall, J. R.; Hartmann, M.; Hollister, E. B.; 
75 (23), 7537-7541. https://doi.org/10.1128/AEM.01541-09.

(10) Westcott, S. L.; Schloss, P. D. OptiClust, an Improved Method for Assigning Amplicon-Based Sequence Data to Operational Taxonomic Units. mSphere 2017, 2

(2), 1-11. https://doi.org/10.1128/mspheredirect.00073-17.

108

(11) Quast, C.; Pruesse, E.; Yilmaz, P.; Gerken, J.; Schweer, T.; Glo, F. O.; Yarza, P. The SILVA Ribosomal RNA Gene Database Project : Improved Data Processing and WebBased Tools. 2013, 41 (November 2012), 590-596.

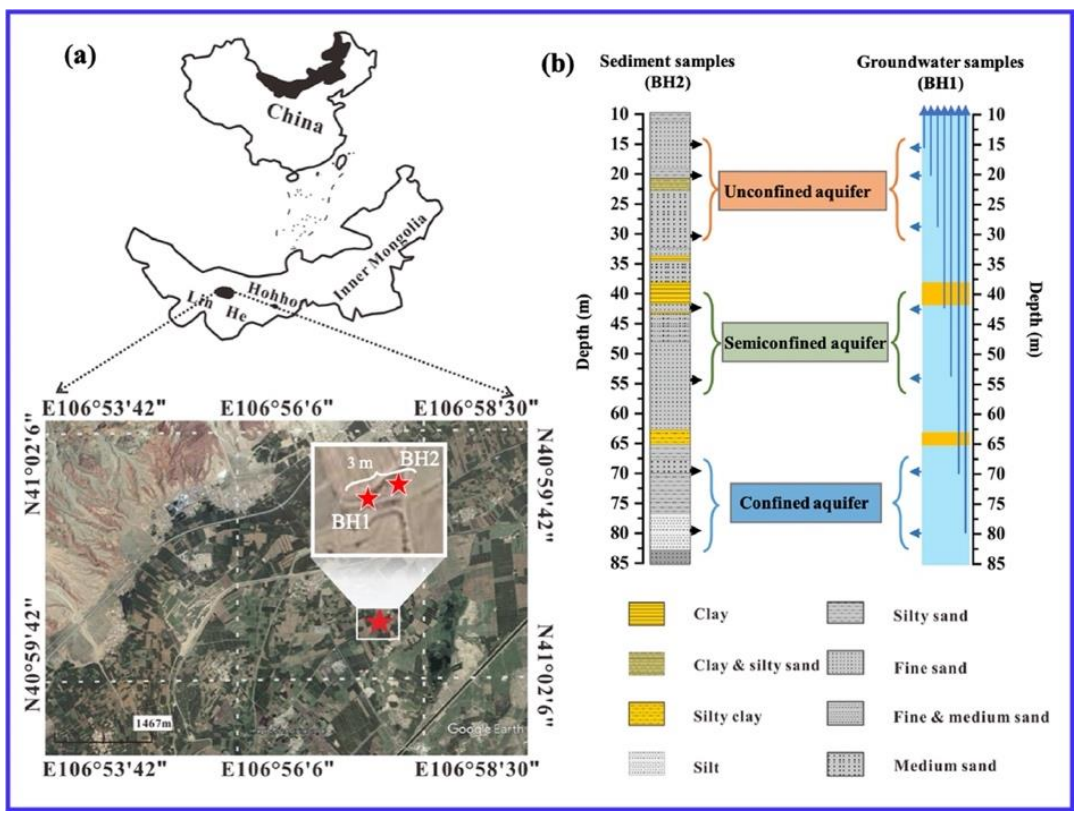

114 Fig. S1 (a) Locations of the study area and groundwater-sediment sampling sites and (b)

115 lithological profiles of $\mathrm{BH} 2$ sediments and multi-level groundwater sampling depths of BH1 116 borehole. 
UnConfined aquifer-1

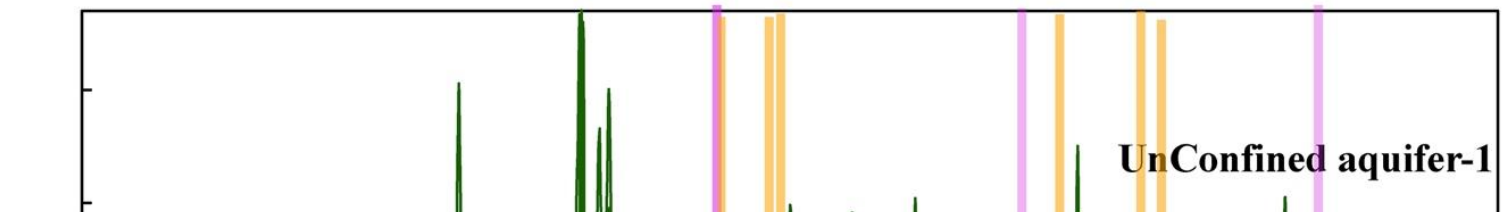

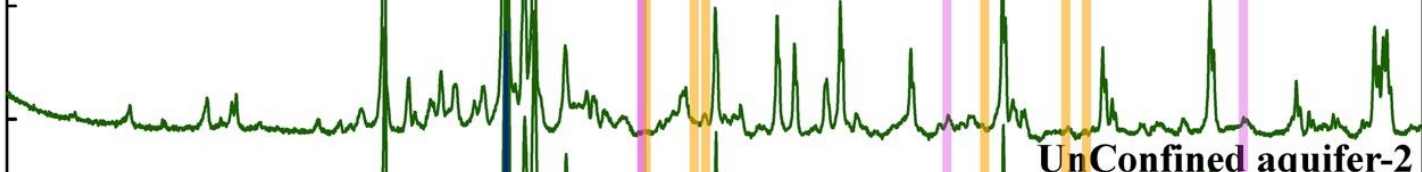
UnConfined aquifer-2

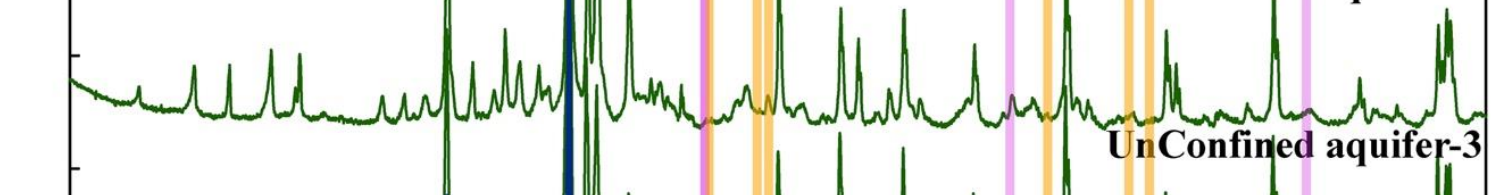

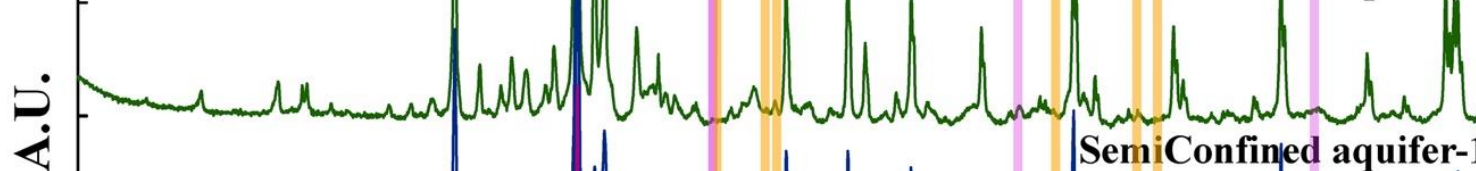
SemiConfined aquifer-1
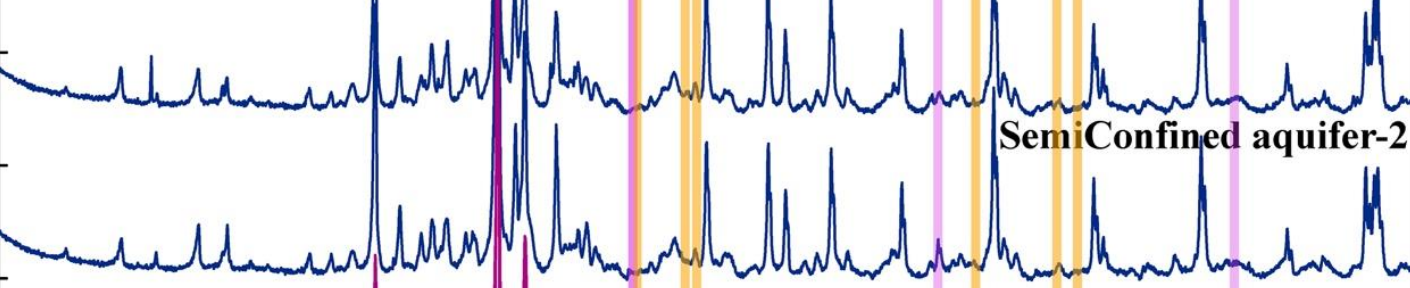
Confined aquifer-1

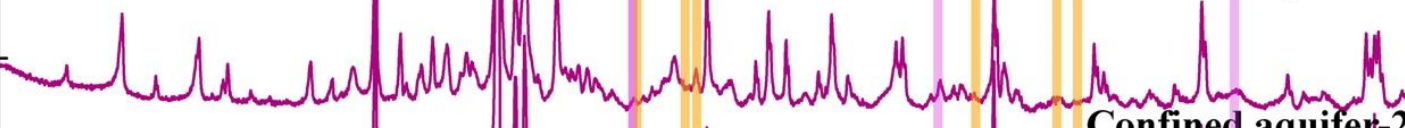

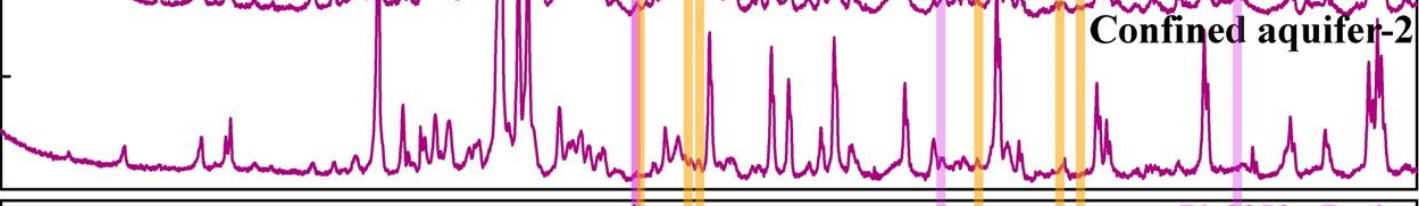

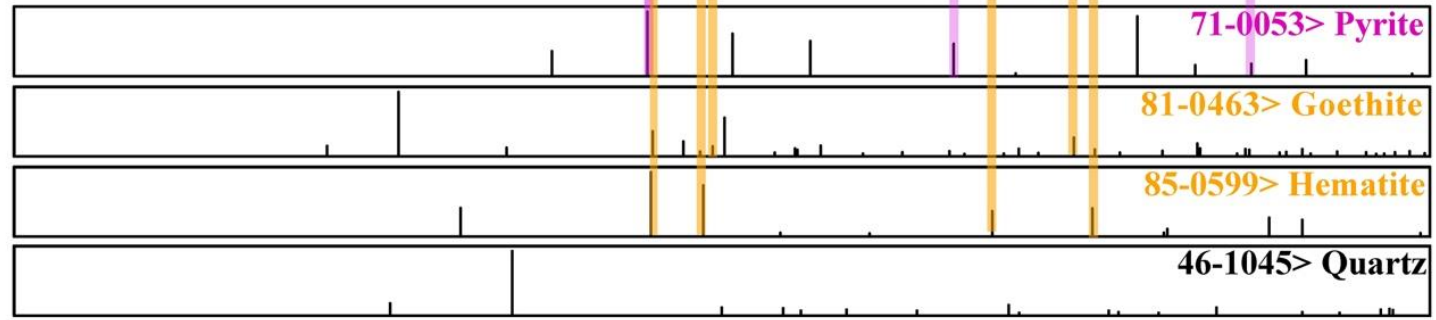
89-6427> Albite

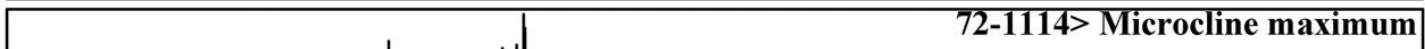

72-1114> Microcline maximum

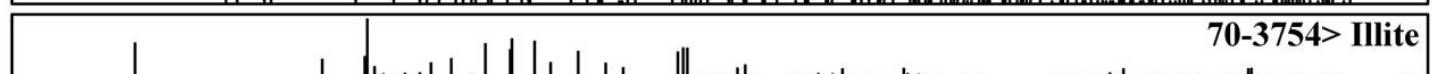
| 83-1380 > Chlorite

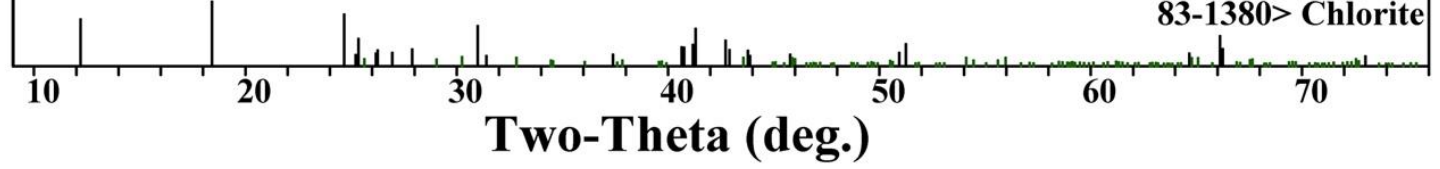

Fig. S2 XRD patterns of the sediment samples from different aquifers. 


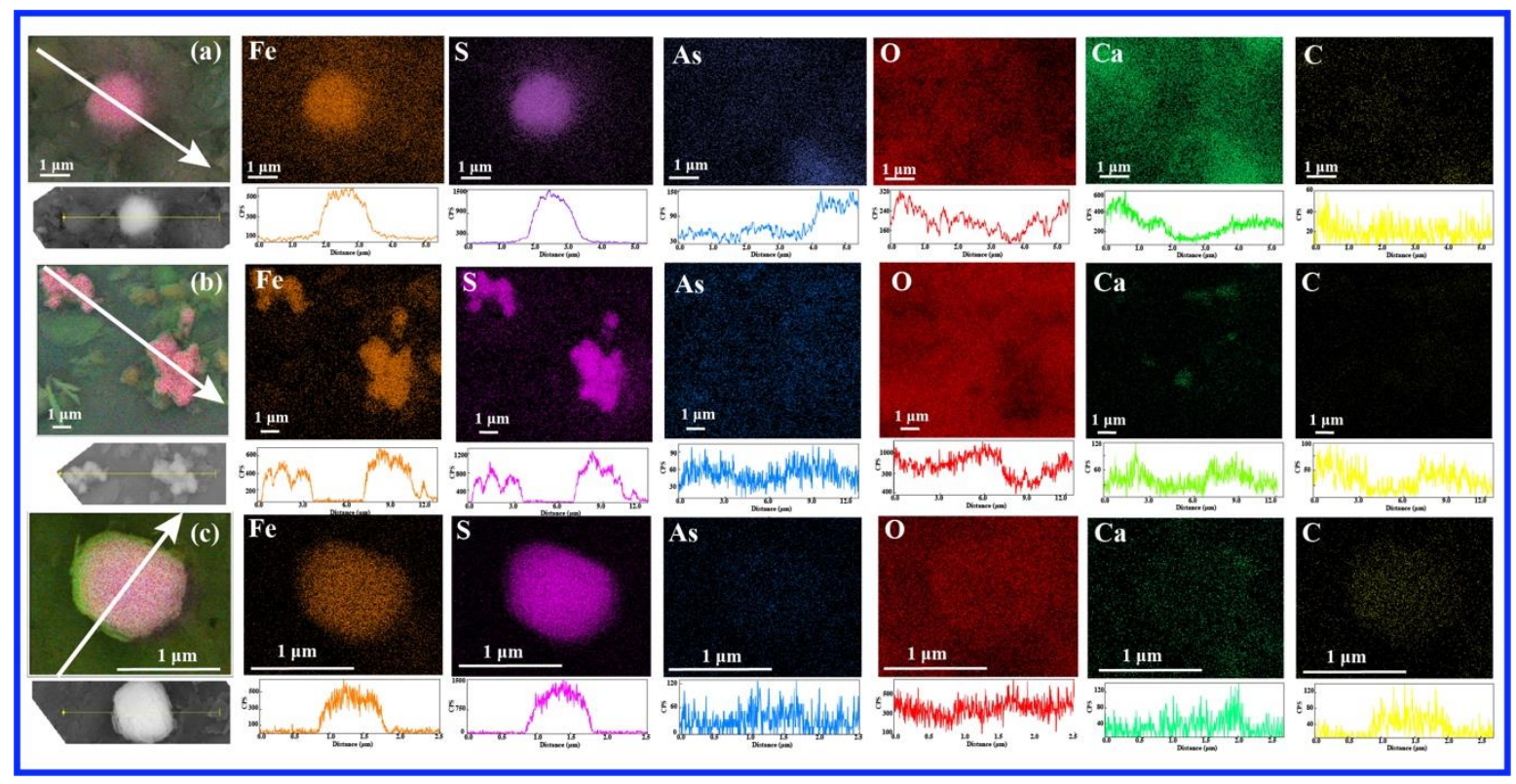

122 Fig. S3 SEM-EDS images of pyrite particles in sediment samples from unconfined (a),

123 semiconfined (b), and confined (c) aquifers. 


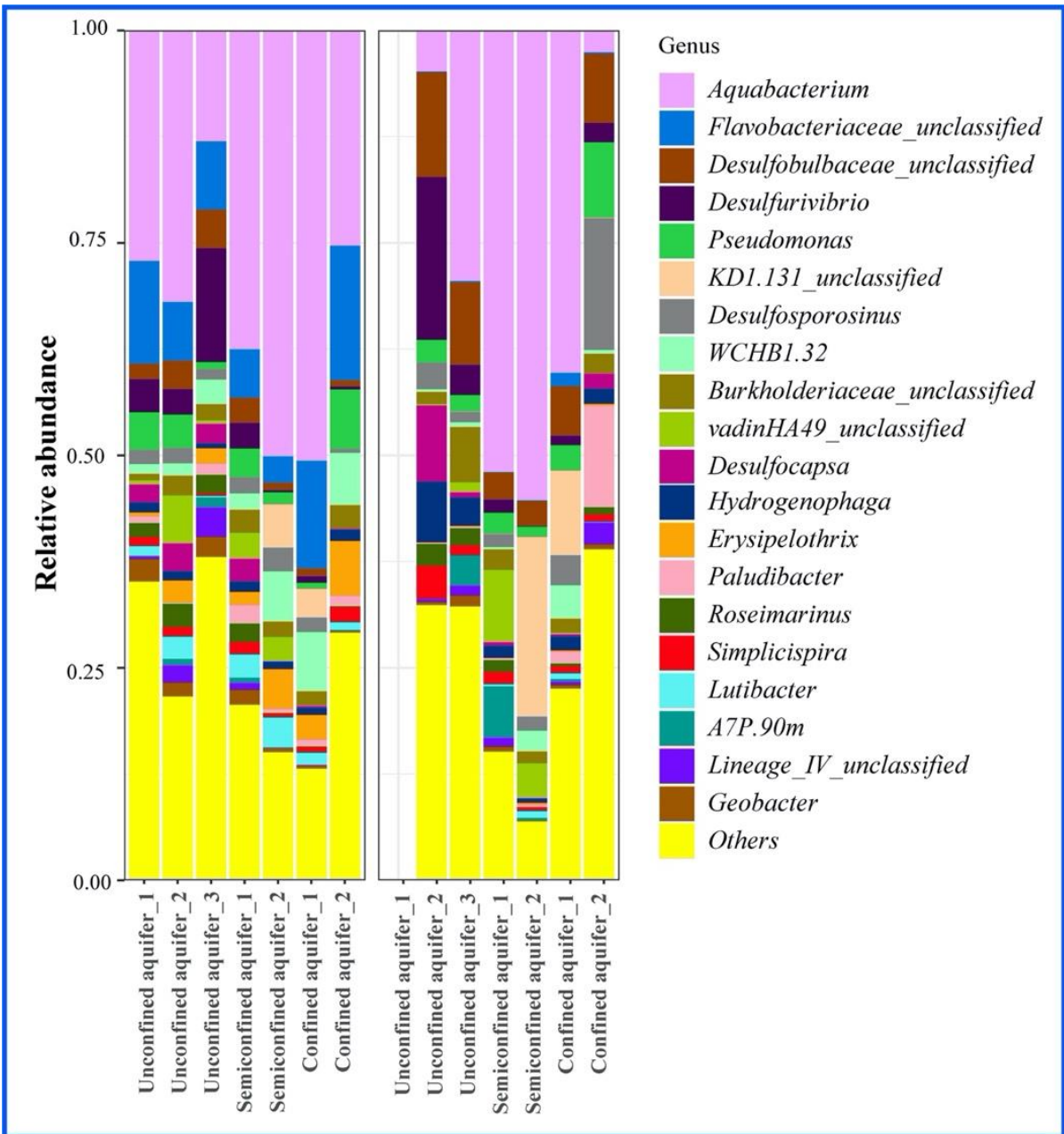

Fig. S4 Groundwater bulk (left) and active (right) microbial communities in different aquifer 128 samples. 


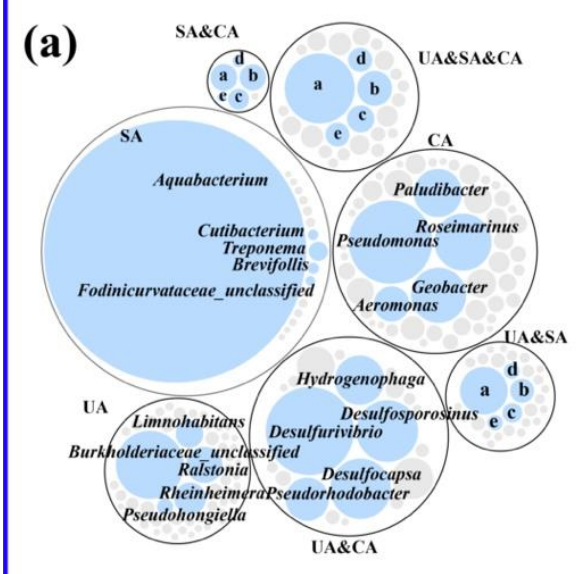

Groundwarter active genera

SA\&CA

a Sulfurospirillum

b Marinilabiliaceae_unclassified

c Oxobacter

d Bacteroidia_unclassified

e Bosea

UA\&SA\&CA

a Desulfobulbaceae_unclassified

b Fibrobacteraceae unclassified

c Rhodocyclaceae_unclassified

e Rhizobiaceae_unclassified

UA\&SA

a Simplicispir

b Clostridium

c Cellvibrio

e Tautropia

(b)

A\&SA

Brevundimonas CA

Stenotrophomonas Kocuria Sediment active gener

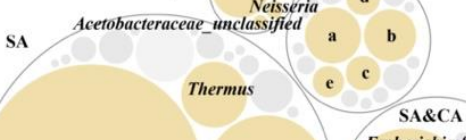

$\begin{array}{lll}\text { Pseudomonas } & \text { Escherichia-Shigella } \\ & \text { Achromobacter }\end{array}$

CA

a Gemmatimonadaceae_unclassified

bimbriimonadaceae_unclassified

d Saccharimonadales unclassified

e Hydrogenophaga

UA\&SA\&CA

a Ralstonia

Rhizobium

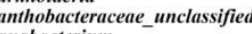

Aquabacterium

UA\&CA

a Sphingomonadaceae_unclassified

b Ruminiclostridium
c Gammaproteobacteria_unclassified

$\begin{array}{ccc}\text { Streptococcus Enhydrobacter } & \text { Acinetobacter }\end{array}$

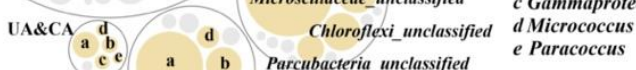

Figure S5 Grouping active genera in groundwater (a) and sediment (b) based on aquifer type,

132 including unconfined aquifer (UA), semiconfined aquifer (SA), and confined aquifer (CA).

133 Light blue and light orange circles indicate the top five active genera that were enriched in

134 groundwater and sediment samples, respectively; and the diameter of both light blue and light

135 orange circles indicate the relative abundance of the active genera. 

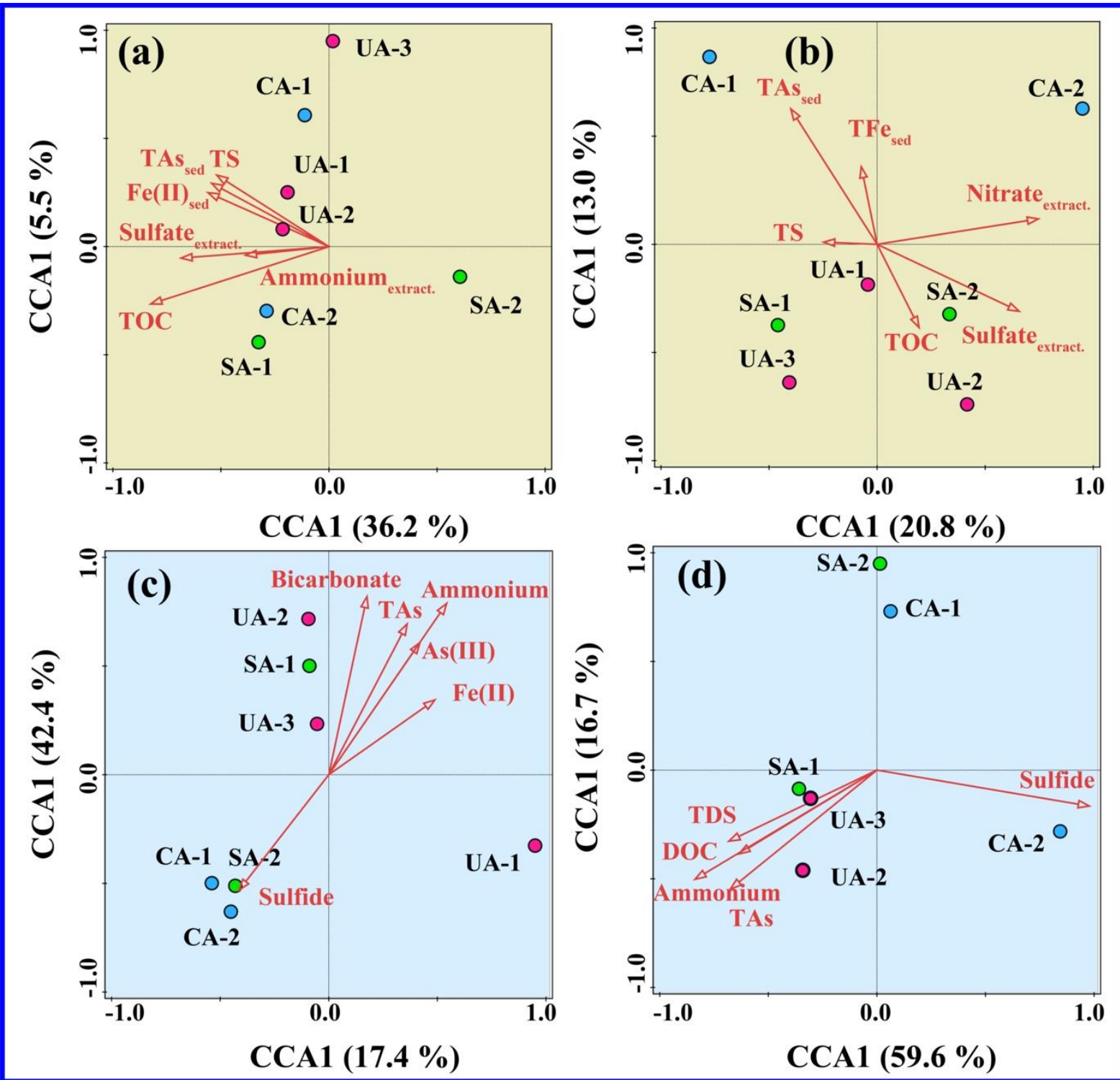

138 Fig. S6 CCA analysis of groundwater bulk (a) and active (b) microbial communities and

139 sediment bulk (c) and active (d) microbial communities. Red, green, and blue circles indicate 140 the samples from unconfined aquifer (UA; Depth BLS: UA-1 < UA-2 < UA-3), semiconfined 141 aquifer (SA; Depth BLS: SA-1 < SA-2), and confined aquifer (CA; Depth BLS: CA-1 < CA142 2), respectively. 


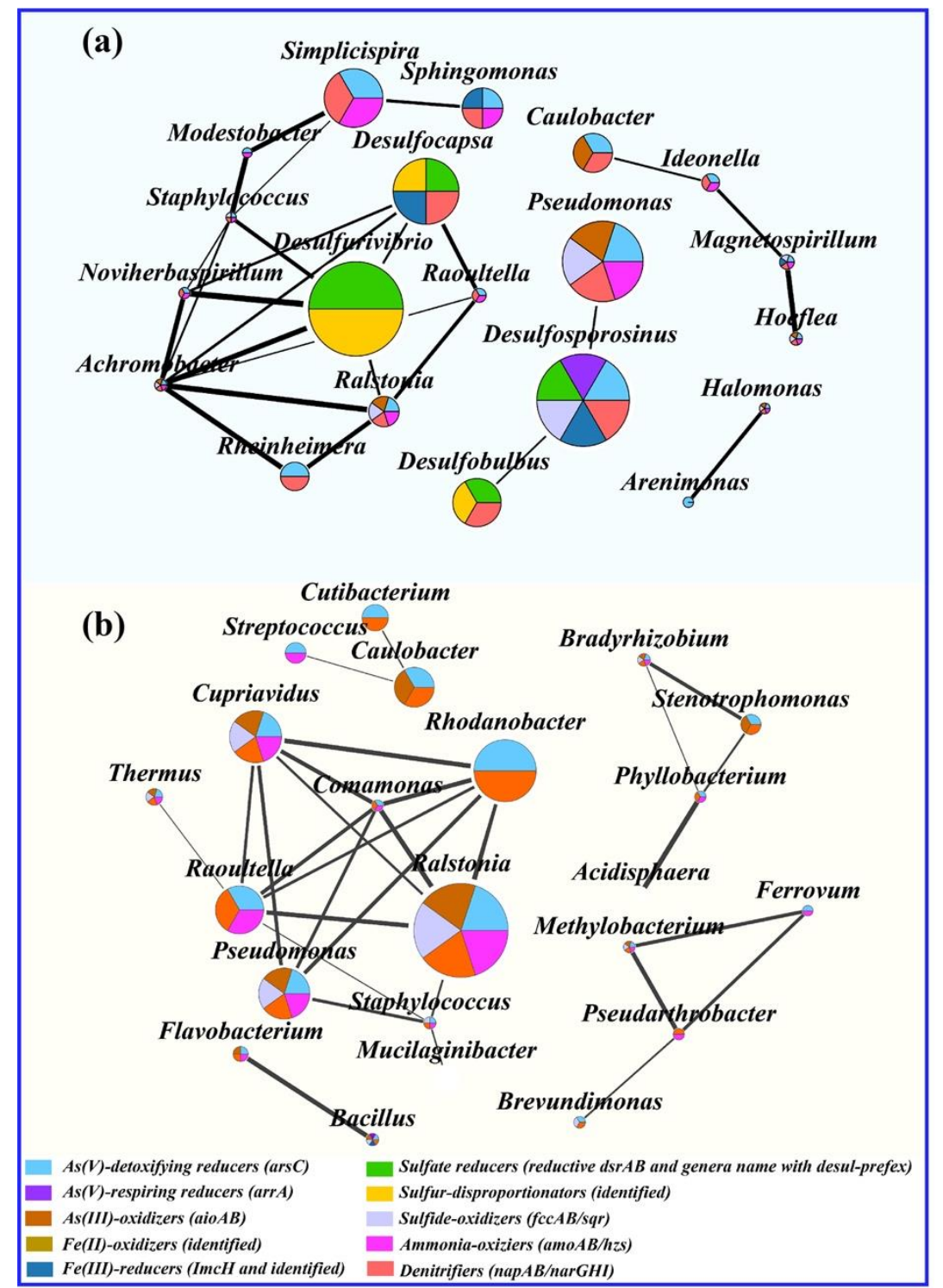

145 Figure S7 Co-occurrence networks of groundwater-soured (a) and sediment-sourced (b) active

146 genera based on Pearson correlations (active in at least three samples). The diameter of the

147 colored circles indicates the average relative abundance in either groundwater or sediment

148 samples (The higher relative abundance is shown with the larger circle), while pie charts inside

149 colored circles indicate the specific As-S-Fe-N metabolic processes that the genera were

150 potentially involved in. The width of the edges between circles indicates the degree of the

151 correlations (the higher correlation is shown with the wider edge). 


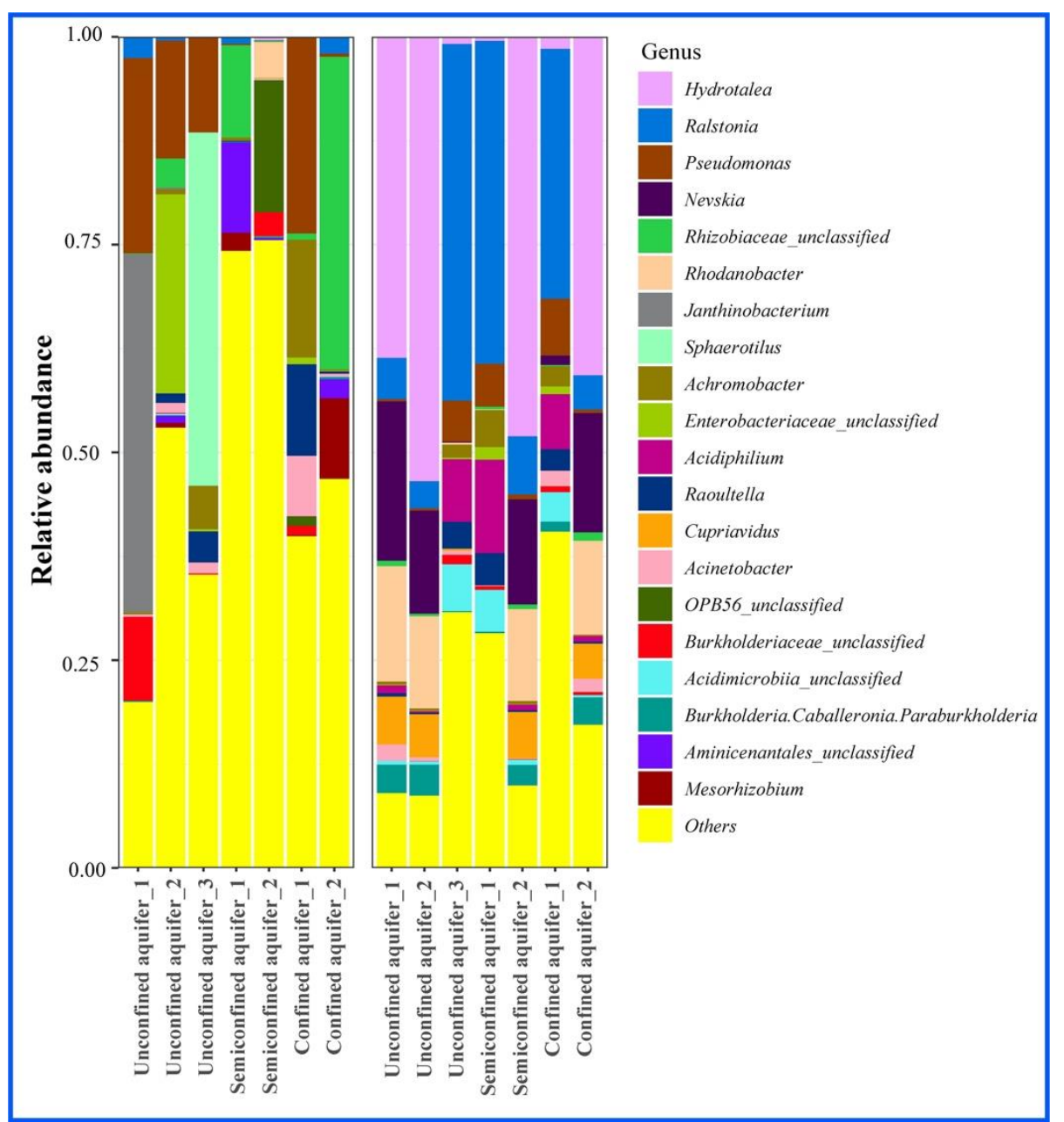

153 Fig. S8 Sediment bulk (left) and active (right) microbial communities in different aquifer samples. 

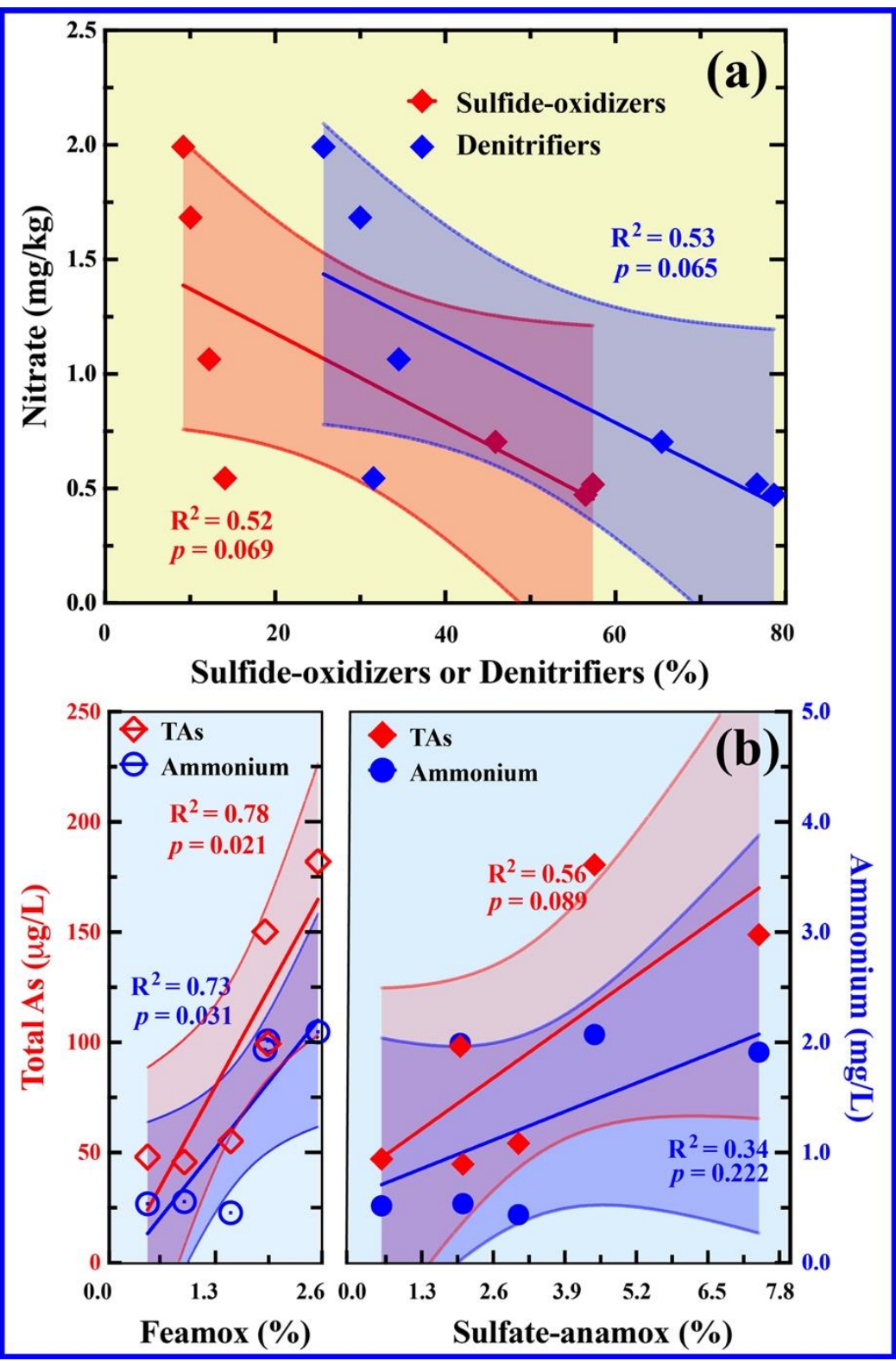

Fig. S9 Linear regression and Pearson correlation analysis: (a) between water extractable

158 nitrate and sulfide-oxidizers and between water extractable nitrate and denitrifiers in sediments;

159 (b) between total dissolved As and Feammox and between ammonium and Feammox (left 160 column) and between total dissolved As and sulfate-anammox (surammox) and between 161 ammonium and sulfate-anammox (surammox) (right column) in groundwaters. 


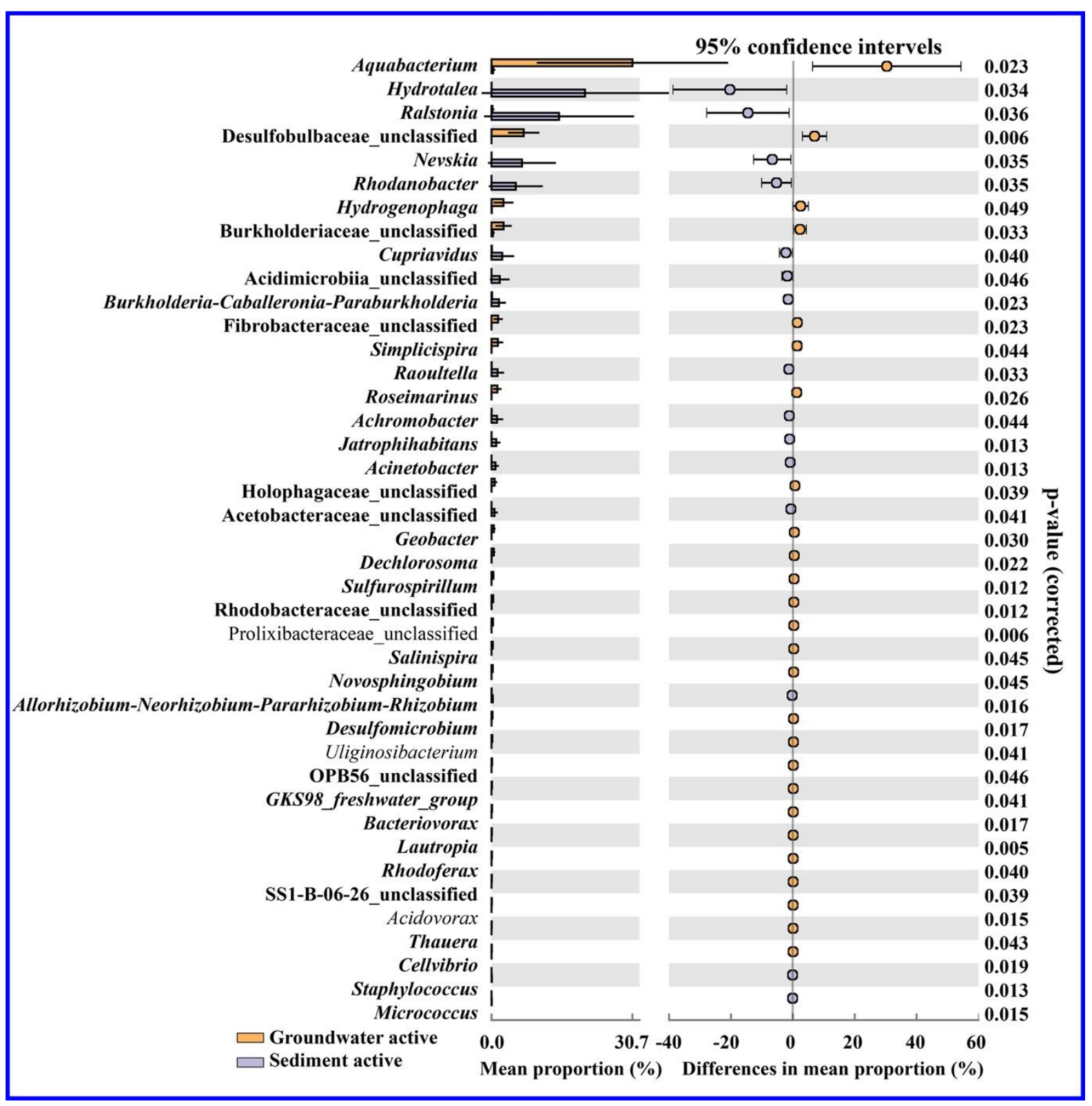

165 Fig. S10 Differences in genera between groundwater and sediment microbial communities

166 based on Wilcoxon rank-sum test (95\% confidence intervals, $p<0.05)$. Active

167 (rRNA/rDNA >1) genera are listed in bold. 
Table S1 Geochemical parameters of sediment and groundwater samples from different aquifers

\begin{tabular}{|c|c|c|c|c|c|c|c|c|c|c|c|}
\hline & & \multicolumn{4}{|c|}{ Unconfined aquifer } & \multicolumn{3}{|c|}{ Semiconfined aquifer } & \multicolumn{3}{|c|}{ Confined aquifer } \\
\hline & & UA-1 & UA-2 & UA-3 & Ave. & UA-1 & UA-2 & Ave. & UA-1 & UA-2 & Ave. \\
\hline \multirow{10}{*}{ Sediment } & TAssed $(\mathrm{mg} / \mathrm{kg})$ & 21.1 & 36.3 & 17.7 & 25.0 & 20.4 & 15.1 & 17.7 & 52.9 & 25.2 & 39.1 \\
\hline & TS $(\mathrm{g} / \mathrm{kg})$ & 19.4 & 61.5 & 35.7 & 38.9 & 26.4 & 16.3 & 21.4 & 45.8 & 26.9 & 36.4 \\
\hline & $\mathrm{TMn}_{\text {sed }}(\mathrm{g} / \mathrm{kg})$ & 2.73 & 3.8 & 1.84 & 2.80 & 2.20 & 1.41 & 1.81 & 4.06 & 2.86 & 3.46 \\
\hline & $\mathrm{Fe}(\mathrm{II})_{\text {sed }}(\mathrm{g} / \mathbf{k g})$ & 9.94 & 16.9 & 6.26 & 11.1 & 7.44 & 5.69 & 6.57 & 17.3 & 9.53 & 13.4 \\
\hline & $\mathrm{TFe}_{\text {sed }}(\mathrm{g} / \mathrm{kg})$ & 15.6 & 31.9 & 10.5 & 19.3 & 14.2 & 10.1 & 12.2 & 27.1 & 18.1 & 22.6 \\
\hline & 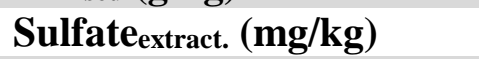 & 471 & 683 & 254 & 469 & 318 & 167 & 242 & 115 & 482 & 298 \\
\hline & Nitrateextract. (mg/kg) & 1.06 & 1.99 & 0.52 & 0.47 & 0.55 & 0.70 & 1.68 & 1.06 & 1.99 & 0.52 \\
\hline & Ammoniumextract. (mg/kg) & 4.82 & 31.8 & 12.9 & 17.1 & 11.0 & 10.0 & 13.6 & 4.82 & 31.8 & 12.9 \\
\hline & TOC (mg/kg) & 111 & 222 & 163 & 165 & 317 & 37.9 & 177 & 47.3 & 194 & 121 \\
\hline & Reflectance $530-520 \mathrm{~nm}$ & 0.44 & 0.36 & 0.35 & 0.38 & 0.39 & 0.20 & 0.30 & 0.25 & 0.21 & 0.23 \\
\hline \multirow[t]{15}{*}{ Groundwater } & Ammonium (mg/L) & 1.80 & 1.92 & 2.08 & 1.93 & 2.00 & 0.52 & 1.26 & 0.54 & 0.44 & 0.49 \\
\hline & Nitrite $(\mu \mathrm{g} / \mathrm{L})$ & 1.00 & 11.0 & 26.0 & 12.7 & 8.00 & 26.0 & 17.0 & 30.0 & 20.0 & 25.0 \\
\hline & $\operatorname{Mn}(\mu \mathrm{g} / \mathrm{L})$ & 254 & 91.0 & 399 & 248 & 144 & 331 & 238 & 143 & 227 & 185 \\
\hline & $\mathrm{TFe}(\mu \mathrm{g} / \mathrm{L})$ & 1.37 & 1.12 & 2.00 & 1.49 & 1.08 & 0.49 & 0.78 & 0.60 & 0.56 & 0.58 \\
\hline & $\mathrm{Fe}(\mathrm{II})(\mu \mathrm{g} / \mathrm{L})$ & 0.83 & 0.37 & 1.20 & 0.80 & 0.84 & 0.36 & 0.60 & 0.24 & 0.20 & 0.22 \\
\hline & Sulfide $(\mu \mathrm{g} / \mathrm{L})$ & 20.0 & 28.0 & 11.0 & 19.7 & 15.0 & 27.0 & 21.0 & 48.0 & 131 & 89.5 \\
\hline & Sulfate (mg/L) & 318 & 268 & 873 & 486 & 772 & 61.4 & 416 & 68.3 & 83.0 & 75.7 \\
\hline & $\operatorname{As}(\mathbf{V})(\mu \mathrm{g} / \mathrm{L})$ & 0.25 & 20.2 & 0.25 & 6.91 & 12.9 & 8.45 & 10.7 & 0.25 & 0.25 & 0.25 \\
\hline & $\operatorname{As}(\mathrm{III})(\mu \mathrm{g} / \mathrm{L})$ & 97.8 & 110 & 148 & 119 & 75.8 & 41.4 & 58.6 & 40.1 & 42.1 & 41.1 \\
\hline & TAs $(\mu \mathrm{g} / \mathrm{L})$ & 115 & 149 & 181 & 148 & 98.6 & 47.3 & 72.9 & 45.0 & 54.5 & 49.8 \\
\hline & Bicarbonate (mg/L) & 338 & 386 & 458 & 394 & 432 & 288 & 360 & 271 & 264 & 268 \\
\hline & DOC $(\mathrm{mg} / \mathrm{L})$ & 1.89 & 2.39 & 3.28 & 2.52 & 3.79 & 1.26 & 2.53 & 2.04 & 1.89 & 1.96 \\
\hline & TDS (g/L) & 1.57 & 1.31 & 3.09 & 1.99 & 2.83 & 0.56 & 1.69 & 0.55 & 0.57 & 0.56 \\
\hline & pH & 7.75 & 7.49 & 7.61 & 7.62 & 8.16 & 8.06 & 8.11 & 7.99 & 7.93 & 7.96 \\
\hline & -ORP (mV) & 81.6 & 100 & 158 & 113 & 145 & 124 & 135 & 153 & 107 & 130 \\
\hline
\end{tabular}


170 Table S2 Alpha diversity of microbial community in sediment and groundwater samples from

171 different aquifers

\begin{tabular}{cccccc}
\hline Matrix & $\begin{array}{c}\text { Extracted } \\
\text { material }\end{array}$ & Aquifer & $\begin{array}{c}\text { Shannon } \\
\text { index }\end{array}$ & $\begin{array}{c}\text { Simpson } \\
\text { index }\end{array}$ & Chao1 \\
\hline Groundwater & DNA & Unconfined & 3.92 & 0.09 & 611 \\
Groundwater & DNA & Semiconfined & 3.06 & 0.21 & 679 \\
Groundwater & DNA & Confined & 3.01 & 0.18 & 505 \\
Groundwater & RNA & Unconfined & 3.97 & 0.07 & 605 \\
Groundwater & RNA & Semiconfined & 2.33 & 0.31 & 578 \\
Groundwater & RNA & Confined & 3.89 & 0.10 & 615 \\
Sediment & DNA & Unconfined & 3.01 & 0.19 & 348 \\
Sediment & DNA & Semiconfined & 4.10 & 0.04 & 459 \\
Sediment & DNA & Confined & 2.92 & 0.17 & 256 \\
Sediment & RNA & Unconfined & 2.38 & 0.22 & 159 \\
Sediment & RNA & Semiconfined & 2.64 & 0.19 & 158 \\
Sediment & RNA & Confined & 3.15 & 0.14 & 251 \\
\hline
\end{tabular}

